\title{
SLAM-based Follow-the-Leader Deployment of Concentric Tube Robots
}

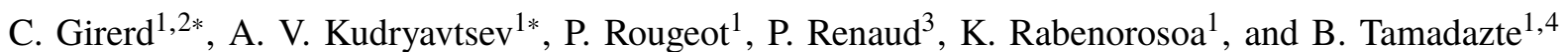

\begin{abstract}
This paper deals with an original combination of navigation by simultaneous localization and mapping (SLAM) and follow-the-leader (FTL) deployment for the control of concentric tube robots (CTRs). The objective is to make possible the automatic navigation of such continuum robots within an anatomical structure, by simultaneously managing both collision avoidance and map updating of the environment, i.e., of the surrounding anatomical structures. Indeed, CTRs designed to achieve a FTL deployment to date were deployed on path identified during a planning task. In this work, this limitation is overcome by embedding, in the control scheme, an online trajectory estimation, therefore allowing an autonomous deployment of CTRs with an approximate FTL behavior. The proposed hybrid control law integrates a stability criterion to ensure at the same time CTR stability during deployment, required for a FTL deployment. The interest of the approach is demonstrated through simulation using realistic anatomical data and experiments, with a 3 degrees-of-freedom CTR.
\end{abstract}

\section{INTRODUCTION}

Navigating within the human body remains challenging when targeted organs are located in confined spaces or are located in a fragile environment. Several robot paradigms have been proposed in order to make such navigation possible: active catheters for vascular navigation and interventions [1], concentric tube robots (CTRs) [2] including designs with embedded actuators [3], hyper-redundant structures such as the $\mathrm{i}^{2}$ Snake [4]. CTRs interestingly offer a high compactness and the ability to deploy along predefined paths, including in a follow-the-leader (FTL) manner [5], [6] where the CTR body follows its tip.

Traditionally, navigation can be achieved with two approaches, either open-loop based on an offline trajectory planning [7] using 3D models reconstructed from medical images [8], or using automatic navigation [9]. Implementation of image-based deployment relies on the use of an external imaging system for an eye-to-hand configuration, or on an eye-in-hand one when a visual sensor is mounted at the tip of the robot. Intracorporeal navigation requires a high degree of accuracy to ensure safety. Image-guided control with eye-in-hand configuration is particularly well-suited for continuum robots [10]: it makes target recognition easier,

This work has been supported by the EIPHI Graduate School (ANR17-EURE-0002) and ANR NEMRO (ANR-14-CE17-0013).

1 Authors are with FEMTO-ST Institute, Univ. Bourgogne FrancheComté, CNRS, Besançon, France. brahim. tamadazte@femto-st.fr

2 Author is with the Department of Mechanical and Aerospace Engineering, University of California, San Diego, La Jolla, CA 92093 USA. cgirerd@eng.ucsd.edu (corresponding author)

3 Author is with ICube, UDS-CNRS-INSA, Strasbourg, France. pierre.renaud@insa-strasbourg.fr

${ }^{4}$ Institute for Intelligent Systems and Robotics, Sorbonne University, CNRS

${ }^{*}$ These authors contributed equally to this work. allows a close visualization of the surgical site, and leads to less registration issues compared to eye-to-hand approaches.

Controlling a CTR with an eye-in-hand configuration was indeed recently demonstrated to provide a high degree of accuracy during positioning tasks with model-based [11] or model-free [10] visual servoing schemes. However, the autonomous navigation of CTRs is still an open challenge [2]. This seems however mandatory given i) the inter-patient variability which can make hazardous CTR control based on a priori knowledge which is not patient specific, ii) the possible anatomy variations between planning and inspection phases, and iii) the existence of physiological motions such as breathing-induced motions, leading to time-varying displacements and deformations of the patient anatomy. Considering the environment of CTRs in real-time during navigation is needed, and can be well illustrated by the task of the olfactory cleft inspection, where contacts with tissues are not admissible according to the clinical requirements [12].

SLAM methods allow a robot to simultaneously compute the sensor pose and update a map of an unknown environment [13], [14]. SLAM has been greatly improved to obtain accurate and robust approaches offering satisfying localization and mapping. In this work, our contribution is to combine state-of-art SLAM with closed-loop control based on an eye-in-hand configuration to make safe autonomous approximate FTL deployment of CTRs in confined spaces. Safety is also considered by checking CTR stability within the controller scheme. To develop and assess this proposition, we consider a 3-tube CTR design, previously identified as relevant for navigation in the nasal cavity [12]. The tube geometries of the robot were selected for FTL deployment using a dataset of nasal cavities. The control law based on visual SLAM allows to approximate the traditional FTL deployment of the CTR without any subject-specific planning task. Simultaneously, the SLAM algorithm provides a reconstruction of the anatomical environment of interest. Such algorithms have been used in combination with CTR [9], but to the best of our knowledge, this is the first exploitation of visual SLAM for the deployment of CTRs.

The paper is organized as follows. Section II introduces required elements related to CTR kinematics as well as the stability criterion included in the developed controller. Section III focuses on the SLAM algorithm and on the formulation of the autonomous navigation controller of a CTR. Validation through simulation using realistic anatomical data thanks to a developed open-source simulator, and experiments, are described in Sections IV and V, respectively. 


\section{CONCENTRIC TUBE ROBOTS: BACKGROUND}

\section{A. Kinematics}

During a deployment in free space, as considered in this work, the kinematics of a CTR composed of $n$ tubes is formulated as a boundary value problem governed by differential algebraic equations [15]. Using the variables and notations reported in Table I, they are expressed as

$$
k_{i t} \ddot{\psi}_{i}=\frac{k_{i b}}{k_{b}} \sum_{j=1}^{n} k_{j b} \kappa_{i} \kappa_{j} \sin \left(\psi_{i}-\psi_{j}\right),
$$

with $i \in\{1, \ldots, n\}$ and $k_{b}=\sum_{i=0}^{n} k_{i b}$. The derivatives are taken with respect to $s$. The tube angles are known at their proximal ends, where they are attached to their actuators, while their torsion equals zero at their free ends (Fig. 1(a), 1(b)). This leads to the boundary conditions (2):

$$
\left\{\begin{array}{l}
\psi_{i}(0)=\psi_{i}\left(\beta_{i}\right)-\beta_{i} \dot{\psi}_{i}(0) \\
\dot{\psi}_{i}\left(L_{i}+\beta_{i}\right)=0
\end{array}\right.
$$

TABLE I

Nomenclature.

\begin{tabular}{ll}
\hline$n$ & Number of tubes in the concentric tube robot \\
$\kappa_{i}$ & Curvature of tube $i$ \\
$\psi_{i}$ & Angle between the material frame of tube $i$ and the Bishop frame \\
$k_{i b}$ & Bending stiffness of tube $i$ \\
$k_{i t}$ & Torsional stiffness of tube $i$ \\
$L_{i}$ & Total length of tube $i$ \\
$\beta_{i}$ & Transmission length of tube $i$ \\
$l_{i}$ & Deployed length of link $i$ of the CTR \\
$\gamma_{i}$ & Curvature of link $i$ of the CTR \\
$\mathbf{p}_{B}$ & Position of the backbone Bishop frame \\
$\mathbf{R}_{B}$ & Rotation matrix of the Bishop frame along the CTR backbone \\
$s$ & Curvilinear abscissa of the CTR \\
$\mathbf{e}_{i}$ & $i-t h$ standard basis vector \\
& Conversion of an element of $\mathbb{R}^{3}$ to an element of $\mathfrak{s D}(3)$ \\
$d_{\pi}$ & Distance between the CTR tip and the plane $\pi$ \\
$\pi$ & Plane normal to the CTR tip, located at a distance $d_{\pi}$ to its tip \\
$\mathcal{H}$ & 3D point cloud given by the SLAM algorithm \\
$\mathcal{X}$ & 2D point cloud on the plane $\pi$ \\
$\epsilon_{F}$ & Filter coefficient for the computation of $F$ \\
$F$ & Repulsive force exerted by $\mathcal{X}$ \\
$\mathbf{p}_{E} E$ & Current 3D position of the end-effector of the CTR \\
$\mathbf{p}_{E E}^{*}$ & Future 3D position of the end-effector of the CTR \\
$\mathbf{J}_{\mathbf{T}}^{\mathbf{i n v}}$ & Jacobian matrix for the "Translating" task \\
$\mathbf{J}_{\mathbf{R}}^{\mathbf{i n v}}$ & Jacobian matrix for the "Rotating" task \\
$\mathbf{J}_{\mathbf{S}}^{\mathbf{i n v}}$ & Specific Jacobian matrix for the "Rotating" task \\
$\lambda_{T}$ & Gain for the "Translating" task \\
$\lambda_{R}$ & Gain for the "Rotating" task \\
$\eta$ & Priority ratio between the "Translating" and "Rotating" tasks \\
\hline &
\end{tabular}

The shape of the robot is determined by integration of Eq. (3), with $\mathbf{p}_{1}$ the position and $\mathbf{R}_{1}$ the orientation of the backbone Bishop frame along the robot, and $\hat{\mathbf{u}}_{B}$ the Bishop

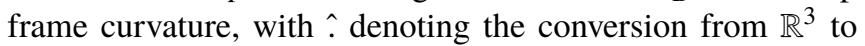
the cross product matrix.

$$
\left\{\begin{array}{l}
\dot{\mathbf{p}}_{1}=\mathbf{R}_{1} \mathbf{e}_{3} \\
\dot{\mathbf{R}}_{1}=\mathbf{R}_{1} \hat{\mathbf{u}}_{B}
\end{array}\right.
$$

Eq (3) are associated to the boundary conditions of Eq (4),

$$
\left\{\begin{array}{l}
\mathbf{p}_{1}(0)=\mathbf{0} \\
\mathbf{R}_{1}(0)=\mathbf{R}_{z}\left(\psi_{1}(0)\right) .
\end{array}\right.
$$

\section{B. Stability Conditions}

CTRs can exhibit instabilities during deployment [16], [17]. Global stability criterion exist only for CTRs made of two constant-curvature tubes. Local stability criterion exist for CTRs made of any number of piecewise constant curvature tubes [16]. The latter is derived from the linearization of the system of equations (1) around the equilibrium configuration to assess. The resulting system has the form

$$
\mathbf{K}_{\mathbf{t}} \dot{\psi}\left(L_{1}+\beta_{1}\right)=\mathbf{W}_{2} \mathbf{K}_{\mathbf{t}} \dot{\psi}(0),
$$

where $\mathbf{K}_{\mathbf{t}}=\operatorname{diag}\left(k_{1 t} \cdots k_{n t}\right)$. The matrix $\mathbf{W}_{2}$ depends on the tube curvatures, deployed and transmission lengths, and on their bending and torsional stiffnesses. A CTR is stable if $\operatorname{det}\left(\mathbf{W}_{2}\right)>0$. As it is a local stability criterion, it must be verified at any time during deployment, with the corresponding deployed lengths.

\section{FTL Deployment}

Situations of FTL deployment were reported in [5], [6], [18]. When the CTR is composed of tubes with constant curvatures, one requirement is to arrange them in a plane, so that they have aligned or opposite curvatures. This is the situation considered in this paper. Only the tube translations are thus used during the CTR deployment. To ensure that the CTR respects a FTL deployment, the condition $\operatorname{det}\left(\mathbf{W}_{2}\right)>0$ must hold so that robot stays in its plane of deployment.

\section{NAVIGATION USING VISUAL SLAM}

Our approach is intended to use SLAM paradigm to ensure CTR deployment. The overall strategy for CTR navigation, summarized in Algorithm 1, is detailed hereafter. In the remaining of the paper, the CTR considered is made of an inner straight tube and two outer curved tubes, that conform to make a straight, curved, and straight links as illustrated in Fig. 2, compatible with a deployment in the nasal cavity which is the clinical motivation of this work [12].

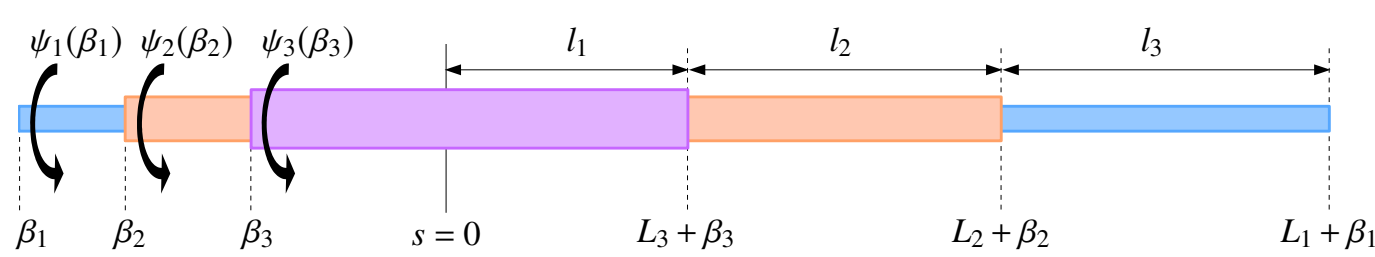

(a)

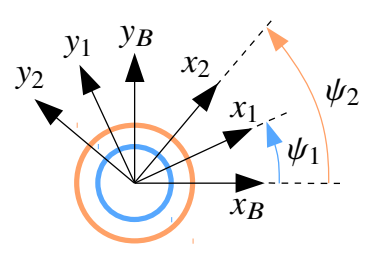

(b)

Fig. 1. (a) 3-tube CTR straightened for ease of understanding, and (b) cross-sectional view of link 2 of the CTR with the orientation of the material base frames of tubes 1 and 2 relatively to the Bishop frame. 


\section{A. Selection of a SLAM Algorithm}

Visual SLAM algorithms are designed to take advantage of the rich information available in the image data. The related literature is varied. Among the recent developments in this field, one can mention the DSO-SLAM approach [19]. It can be considered as an intermediate method between purely sparse methods such as ORB-SLAM [20] and dense approaches such as DTAM [21]. In this work, we opt for the DSO-SLAM algorithm for three main reasons. First, purely sparse methods are very sensitive to the illumination changes and motion blur. Second, dense approaches generally require a GPU implementation and associated powerful hardware. Third, as reported in [22], DSO-SLAM is more accurate than both ORB-SLAM [20] and LSD-SLAM [23] in camera pose estimation, thanks to the combination of a direct probabilistic model which minimizes a photometric error, with a consistent joint optimization of all model parameters.

\section{B. Task 1: Translating}

Each tube is only actuated in translation, so the joint vector is $\mathbf{q}^{\top}=\left(\begin{array}{lll}L_{1}+\beta_{1} & L_{2}+\beta_{2} & L_{3}+\beta_{3}\end{array}\right)$. While moving in the plane, the end-effector has 3 degrees-of-freedom (DOF) (Fig. 2): translations along $\mathbf{x}_{\mathbf{E E}}$ and $\mathbf{z}_{\mathbf{E E}}$ axes, and one rotation $\theta$ about the $\mathbf{y}_{\mathbf{E E}}$ axis. For the CTR deployment, the primary task is to move along the optical axis of the camera that coincides with $\mathbf{z}_{\mathbf{E E}}$, i.e., the axis of the end-effector. The last link composed by the inner tube is straight, so for this task referred to with the index $T$, only the inner tube is actuated with velocity $v$, and the inverse kinematic model is:

$$
\dot{\mathbf{q}}_{\mathbf{T}}=\left(\begin{array}{lll}
0 & 1 & 0 \\
0 & 0 & 0 \\
0 & 0 & 0
\end{array}\right)\left(\begin{array}{l}
0 \\
v \\
0
\end{array}\right)=\mathbf{J}_{\mathbf{T}}^{\mathbf{i n v}}\left(\begin{array}{l}
0 \\
v \\
0
\end{array}\right) .
$$

\section{Task 2: Rotating}

Obviously, CTR tip translation is not sufficient. Due to the curved nature of intracorporeal passageways, rotations

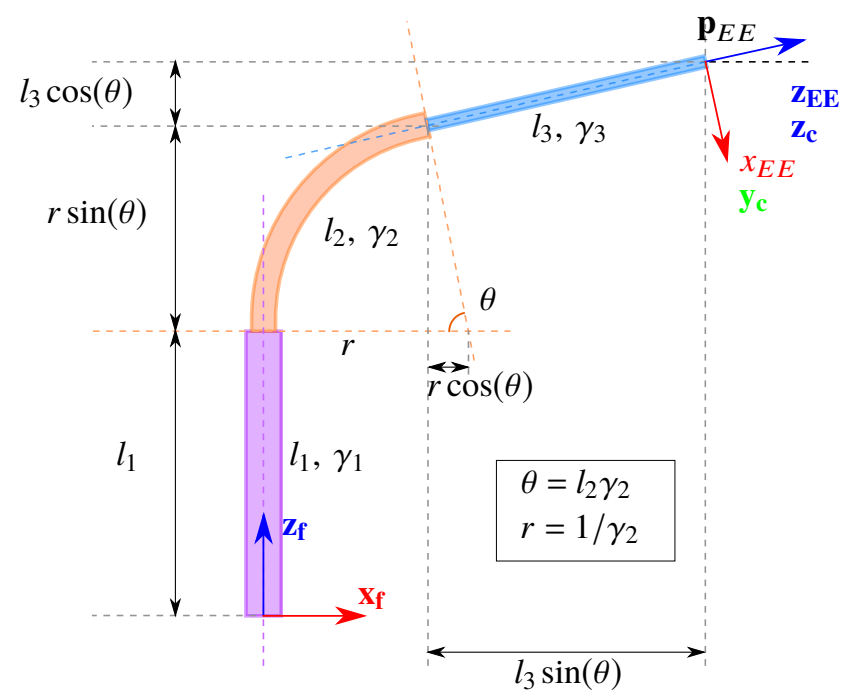

Fig. 2. Geometry of the CTR under consideration. $l_{i}$ and $\gamma_{i}$ are the length and curvature of $i$-th link of the CTR. around its tip are needed to change the deployment direction, and avoid obstacles faced by the robot. The CTR tip corresponds to the camera center in the eye-in-hand configuration. For this task, designated by the index $R$, the end-effector pose is formulated as a function of link lengths. Thereby, using the geometric relations reported in Fig. 2, we obtain:

$$
\left(\begin{array}{c}
x_{E E} \\
z_{E E} \\
\theta
\end{array}\right)=\left(\begin{array}{c}
r-r \cos (\theta)+l_{3} \sin (\theta) \\
l_{1}+r \sin (\theta)+l_{3} \cos (\theta) \\
l_{2} / r
\end{array}\right),
$$

where $l_{i}$ is the length of the $i$-th link of the CTR, and $r, \theta$ are the curvature radius and the arc angle of the second link. Thus, the analytical Jacobian $\mathbf{J}_{\mathbf{R}}^{\mathbf{i n v}}$ is calculated as follows:

$\mathbf{J}_{\mathbf{R}}^{\text {inv }}=\left(\begin{array}{lll}\frac{\partial x_{E E}}{\partial l_{1}} & \frac{\partial x_{E E}}{\partial l_{2}} & \frac{\partial x_{E E}}{\partial l_{3}} \\ \frac{\partial z_{E E}}{\partial l_{1}} & \frac{\partial z_{E E}}{\partial l_{2}} & \frac{\partial z_{E E}}{\partial l_{3}} \\ \frac{\partial \theta}{\partial l_{1}} & \frac{\partial \theta}{\partial l_{2}} & \frac{\partial \theta}{\partial l_{3}}\end{array}\right)^{-1}=\left(\begin{array}{ccc}\frac{-1}{\tan \left(\gamma_{2} l_{2}\right)} & 1 & \frac{l_{3}}{\sin \left(\gamma_{2} l_{2}\right)} \\ 0 & 0 & \frac{1}{\gamma_{2}} \\ \frac{1}{\sin \left(\gamma_{2} l_{2}\right)} & 0 & \frac{-1}{\gamma_{2}}+\frac{l_{3}}{\tan \left(\gamma_{2} l_{2}\right)}\end{array}\right)$.

The Jacobian $\mathbf{J}_{\mathbf{R}}^{\text {inv }}$ is expressed as a function of link lengths, and not in the joint variables. As introduced in [15], those are related by a so-called specific Jacobian matrix, which takes here the expression:

$$
\dot{\mathbf{q}}=\left(\begin{array}{lll}
0 & 0 & 1 \\
0 & 1 & 1 \\
1 & 1 & 1
\end{array}\right)\left(\begin{array}{l}
\dot{l}_{1} \\
\dot{l}_{2} \\
\dot{l}_{3}
\end{array}\right)=\mathbf{J}_{\mathbf{S}}^{\mathbf{i n v}}\left(\begin{array}{l}
\dot{l}_{1} \\
\dot{l}_{2} \\
\dot{l}_{3}
\end{array}\right) .
$$

The "Rotating" task, $\dot{\mathbf{q}}_{\mathbf{R}}$ is then expressed as:

$$
\dot{\mathbf{q}}_{\mathbf{R}}=\mathbf{J}_{\mathbf{S}}^{\text {inv }} \mathbf{J}_{\mathbf{R}}^{\text {inv }}\left(\begin{array}{l}
0 \\
0 \\
\omega
\end{array}\right),
$$

where $\omega$ is the angular velocity. All the tube translations are required to perform this task.

\section{Combining the Tasks}

The activation of the rotating task and its direction depend on the environment. The robot will indeed start rotating when its tip gets closer to an obstacle. The switching mechanism between the two tasks is developed hereafter in five main steps. We first introduce the following notations:

- $\mathcal{H}=\left[\mathbf{h}_{1} \mathbf{h}_{2} \cdots \mathbf{h}_{N}\right]$ is the set of 3D points given by the SLAM algorithm, that corresponds to the estimation of the surface of the environment;

- $\mathcal{X}=\left[\mathbf{x}_{1} \mathbf{x}_{2} \cdots \mathbf{x}_{M}\right]$ is a projection of points of $\mathcal{H}$ that are close to the future tip position;

- $\mathbf{p}_{E E} \in \mathbb{R}^{3}$ is the current 3D position of the end-effector;

- $\mathbf{p}_{E E}^{*} \in \mathbb{R}^{3}$ is the future 3D position of the end-effector.

Step 1: Determination of plane $\pi$ : As a first step, a plane $\pi$ is built perpendicular to the optical axis of the camera and located at a distance $d_{\pi}$ from the end-effector (Fig. 3). The normalized vector $\mathbf{z}_{\mathbf{c}}$ corresponds to the camera optical axis. The point $\mathbf{p}_{E E}^{*}$ can then be expressed as:

$$
\mathbf{p}_{E E}^{*}=\mathbf{p}_{E E}+d_{\pi} \mathbf{z}_{\mathbf{c}},
$$

and the plane $\boldsymbol{\pi}$ is defined through the normal $\mathbf{z}_{\mathbf{c}}$ and the point $\mathbf{p}_{E E}^{*}$ :

$$
\boldsymbol{\pi}:\left\{\mathbf{p} \in \mathbb{R}^{3} \quad \mid \quad \mathbf{z}_{c}^{\top} \cdot\left(\mathbf{p}-\mathbf{p}_{E E}^{*}\right)=0\right\} .
$$


Step 2: Determination of $\mathcal{X}$ : All the 3D points $\mathbf{h} \in \mathcal{H}$ that are located at a distance lower than $d_{\pi}^{*}$ to $\pi$ are projected on it. A set of 2D points $\mathcal{X}$ is obtained, as visible in Fig. 3. We define a constraint for the future position of the end-effector $\mathbf{p}_{E E}^{*}$ as follows: there should be no physical contact between the robot and the environment. Thus, the distance between $\mathcal{X}$ (and thus $\mathcal{H}$ ) and $\mathbf{p}_{E E}^{*}$ should be superior to $d_{\mathcal{X}}^{\min }$. This leads to the following expression for $\mathcal{X}$ :

$$
\begin{aligned}
& \mathcal{X}:\left\{\mathbf{h} \in \mathcal{H} \quad \mid \quad \operatorname{abs}\left(\mathbf{z}_{c}^{\top} \cdot\left(\mathbf{h}-\mathbf{p}_{E E}^{*}\right)\right)<d_{\pi}^{*} \quad\right. \text { and } \\
&\left.\left\|\left(\mathbf{h}-\mathbf{p}_{E E}^{*}\right) \wedge \mathbf{z}_{c}\right\|>d_{\mathcal{X}}^{\min }\right\} .
\end{aligned}
$$

$\mathcal{X}$ is thus an approximation of the object contour at the future desired position $\mathbf{p}_{E E}^{*}$ of the end-effector.

Step 3: Determination of the repulsive force F: To ensure that the robot tip remains inside the object contour, a repulsive force $F$ is generated [24], [25]. It is similar to the one treated by Coulomb's law: each point of $\mathcal{X}$ exerts a force on the point $\mathbf{p}_{E E}^{*}$ that is inversely proportional to the square of the distance between them and $\mathbf{p}_{E E}^{*}$, so that they repel the tip of the robot (Eq. (14)). This is illustrated in Fig. 4. As only planar motions of the CTR are considered, the projection $F$ of the repulsive force onto $\mathbf{y}_{\mathbf{c}}$ is used (Fig. 3).

$$
F= \begin{cases}\frac{1}{\operatorname{Card}(\mathcal{X})} \sum_{j=1}^{\operatorname{Card}(\mathcal{X})} \mathbf{y}_{\mathbf{c}}^{\boldsymbol{\top}} \frac{\mathbf{x}_{\mathbf{j}}-\mathbf{p}_{E E}^{*}}{\left\|\mathbf{x}_{\mathbf{j}}-\mathbf{p}_{E E}^{*}\right\|^{3}} & \text { if } \operatorname{Card}(\mathcal{X})>0 \\ 0 & \text { otherwise }\end{cases}
$$

Step 4: Filtering of $F$ : The point cloud reconstructed with the SLAM algorithm can be sensitive to image noise or other external disturbances. The computation of the repulsive force is thus alike. Consequently, we filter it using a moving median filter. The force at the $t$-th moment of time is then given by Eq. (15) where $\delta$ is the filtering coefficient:

$$
F_{t}=\delta F_{t-1}+(1-\delta) F \text {. }
$$

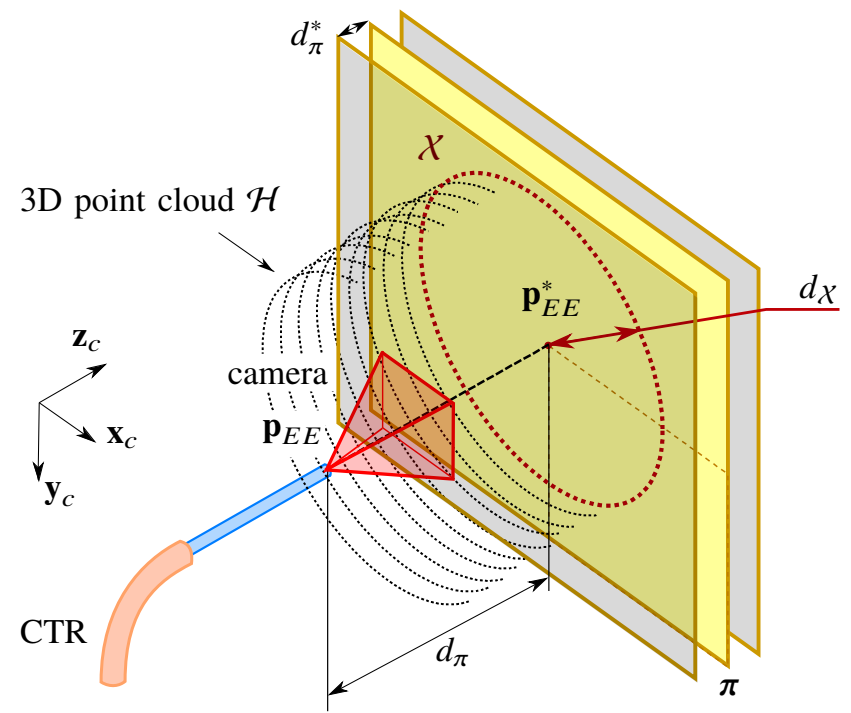

Fig. 3. The points of $\mathcal{H}$ that are closer than $d_{\pi}^{*}$ to the plane $\pi$ are projected on it. The resulting set of $2 \mathrm{D}$ points $\mathcal{X}$ is used for motion planning.

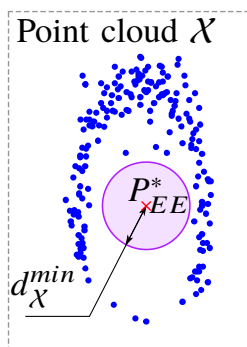

(a)

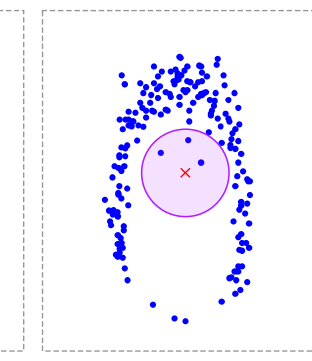

(b)

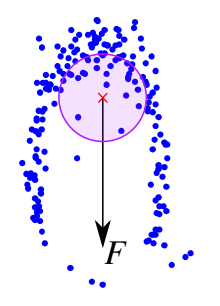

(c)
Fig. 4. Computation of the repulsive force used as a switch between the "Translating" and "Rotating" tasks. In (a), the detected contour of the environment along with the circle of radius $d_{\mathcal{X}}^{\text {min }}$ are represented. In (b), some points of the contour move inside the circle, creating a repulsive force visible in (c).

Step 5: Combination of tasks: Depending on the repulsive force $F$, the implication of $\dot{\mathbf{q}}_{\mathbf{T}}$ for the "Translating" task and $\dot{\mathbf{q}}_{\mathbf{R}}$ for the "Rotating" task differs during the navigation. Thus, let us introduce a parameter $\eta=f(F)$, so that

$$
\dot{\mathbf{q}}=\underbrace{\lambda_{T} \eta \dot{\mathbf{q}}_{\mathbf{T}}}_{\text {Translating }}+\underbrace{\lambda_{R}(1-\eta) \dot{\mathbf{q}}_{\mathbf{R}}}_{\text {Rotating }}
$$

where $\lambda_{T}$ and $\lambda_{R}$ are the task gains. The parameter $\eta$ lies in the interval $[0.5,1]$. When it equals 0.5 , the same priority is assigned to the "Translating" and the "Rotating" tasks. In the extreme case where it equals 1 , only the "Translating" task is activated. The chosen mathematical function enabling such possibility is a sigmoid. In this work, we use the formulation given in (17):

$$
\eta=1.5-\frac{1}{1+e^{-a|F|}},
$$

with $a$ a scalar that controls the slope of the function. Thus, the final expression for the designed control law is:

$$
\dot{\mathbf{q}}=\lambda_{T} \eta \mathbf{J}_{\mathbf{T}}^{\mathbf{i n v}}\left(\begin{array}{l}
0 \\
v \\
0
\end{array}\right)+\lambda_{R}(1-\eta) \mathbf{J}_{\mathbf{S}}^{\mathbf{i n v}} \mathbf{J}_{\mathbf{R}}^{\mathbf{i n v}}\left(\begin{array}{c}
0 \\
0 \\
\operatorname{sign}(F)
\end{array}\right),
$$

where $\omega=\operatorname{sign}(F)$ is used for the angular velocity.

\section{E. Stability Assessment During Deployment}

Stability conditions during deployment are ensured by integrating the criterion presented in section II-B. An analytical expression of $\operatorname{det}\left(\mathbf{W}_{2}\right)$ is available for the CTR geometry under consideration [26]:

$$
\operatorname{det}\left(\boldsymbol{W}_{\mathbf{2}}\right)=\cosh \left(l_{1} \alpha\right)-\alpha \sinh \left(l_{1} \alpha\right)\left(\frac{\beta_{2} k_{3 t}+\beta_{3} k_{2 t}}{k_{2 t}+k_{3 t}}\right),
$$

with

$$
\alpha=\sqrt{-\kappa_{2} \kappa_{3} \frac{k_{3 b} k_{2 b}\left(k_{2 t}+k_{3 t}\right)}{\left(k_{1 b}+k_{2 b}+k_{3 b}\right) k_{2 t} k_{3 t}}} .
$$

Equation (19) is evaluated during deployment. The CTR navigation is stopped if $\operatorname{det}\left(\boldsymbol{W}_{2}\right)<\epsilon$, with $\epsilon>0 . \operatorname{det}\left(\mathbf{W}_{2}\right)$ varies in a smooth way as linear actuation values are smoothly varied [16]. The margin $\epsilon$ therefore ensures that the deployment can be stopped before the robot is unstable, i.e., $\operatorname{det}\left(\boldsymbol{W}_{2}\right)<0$. 


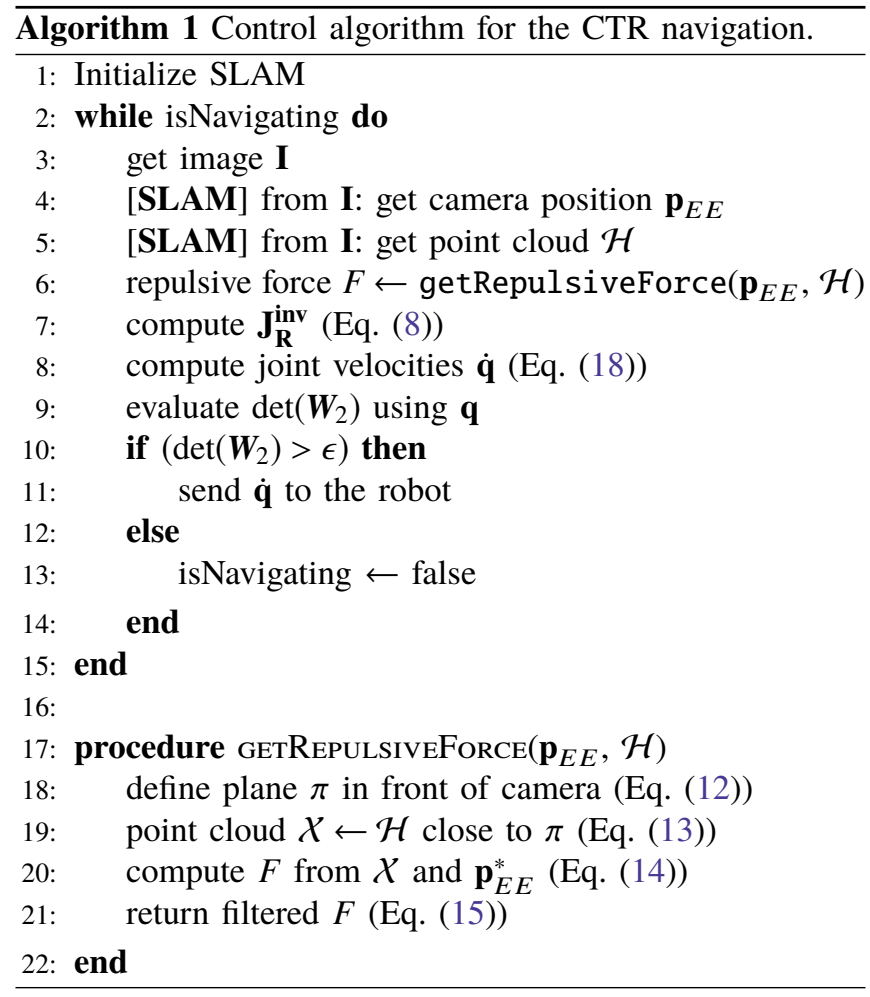

\section{VALIDATION BY SIMULATION}

\section{A. Developed Simulator}

A realistic, generic and open-source simulator is developed specifically for validations ${ }^{1}$. It implements the CTR whose parameters were described previously, as well as a face and a nasal cavity given the proposed application. The 3D models are loaded from STL files, built from CT scans of subjects to reflect the true shape of the nasal corridor. Finally, at the tip of the robot, a virtual camera is simulated with the following intrinsic parameters: focal length $\left(\alpha_{x}, \alpha_{y}\right)=$ $(856.145,856.145)$ pixels, principal point $\left(u_{0}, u_{0}\right)=(320$, 240) pixels, which provides images in PNG format at the resolution of $640 \times 480$ pixels. A screenshot of the developed simulator is depicted in Fig 5.

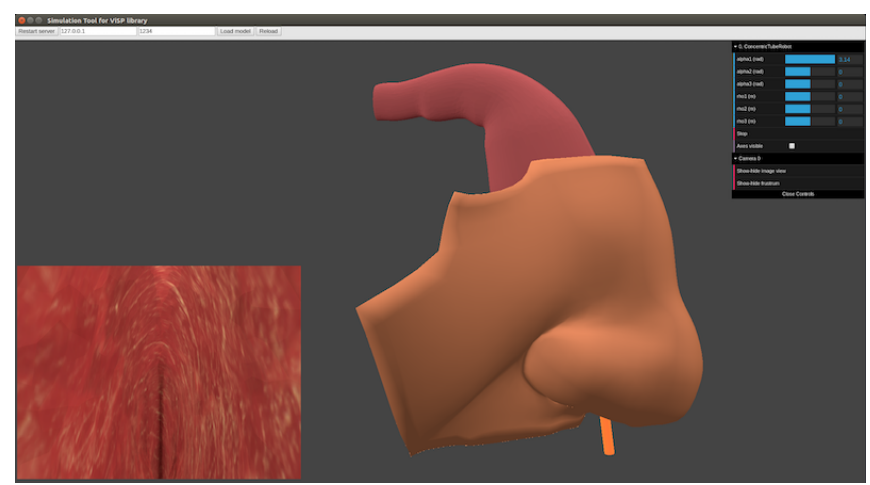

Fig. 5. Snapshot of the developed simulator. The camera image is displayed at the bottom-left in the snapshot.

${ }^{1}$ avkudr.github.io/visa-model-ctr

\section{B. Simulation Results}

In order to evaluate the feasibility of the proposed approach that combines SLAM and FTL deployment, the developed simulator was used. It was configured to reproduce experimental conditions, i.e., the number, length and curvature of the tubes, the camera parameters, and the environment of navigation. This investigation by simulation allows to assess the performance of both methods and materials without, for instance, taking camera limitations into account. The parameters selected for the simulation are $\lambda_{T}=1, \lambda_{R}=1$, $v=0.25 \mathrm{~mm} . \mathrm{s}^{-1}, d_{\pi}=3 \mathrm{~mm}, d_{\pi}^{*}=0.4 \mathrm{~mm}, d_{\mathcal{X}}^{\min }=2 \mathrm{~mm}$, $\delta=0.95, a=10$ and $\epsilon=0.1$. The characteristics of the tubes of the CTR are visible in Table II. The automatic navigation starts with the tubes fully retracted in the actuation unit. They are then deployed in the nasal cavity through the nostril as illustrated in Fig. 5. The tip of the robot, with the camera placed on it, is located under the nostril of the virtual subject. The controller then deploys the robot in accordance with Algorithm 1, with an initialization step that consists in moving the tip of the robot forward to initialize the SLAM algorithm. The results presented in the following are illustrated in the provided video.

Fig. 7 shows that the robot successfully goes through the nasal cavity, while simultaneously estimating the geometry of the environment. The red curve represents the path taken by the camera during navigation relatively to the point cloud. The latter is the complete set of key-points detected by the SLAM algorithm on successive images. An example of keypoints detected on a single frame is visible at the bottom-left illustration of Fig. 7, with the bottom-right image showing the same frame without the key-points. The values of $\eta$ and $F$ over time are visible in Fig. 6a. The deployed lengths of the tubes are also represented in Fig. 6b. As visible in these figures, only the "Translating" task is active during deployment step 1, at the entry of the nasal cavity. The tasks "Translating" and "Rotating" are then active during deployment step 2 to avoid the curved part of the nasal cavity, and then only the "Translating" task is active during deployment step 3 in the olfactory cleft. As shown in Fig.6c, the robot remains stable during deployment, which allows the simulation to be fully conducted. Finally, Fig. 8 shows the superposition of all intermediate configurations adopted

TABLE II

PARAMETERS For CTR DEPLOYMENT SIMULATION.

\begin{tabular}{lccc}
\hline Tube index & 1 & 2 & 3 \\
\hline Material & Nitinol & Nitinol & Steel \\
Inner diam. $(\mathrm{mm})$ & 0.610 & 0.770 & 1.100 \\
Outer diam. $(\mathrm{mm})$ & 0.650 & 1.010 & 1.600 \\
Curvature $\left(\mathrm{mm}^{-1}\right)$ & 0.0000 & 0.0423 & 0.0023 \\
Total length $(\mathrm{mm})$ & 300.0 & 143.0 & 60.4 \\
\hline Links curvature: & & & \\
$\gamma_{1}=0.0000 \mathrm{~mm}^{-1}$ & $\circ$ & 0 & 0 \\
$\gamma_{2}=0.0400 \mathrm{~mm}^{-1}$ & $\circ$ & 0 & \\
$\gamma_{3}=0.0000 \mathrm{~mm}^{-1}$ & $\circ$ & & \\
\hline
\end{tabular}




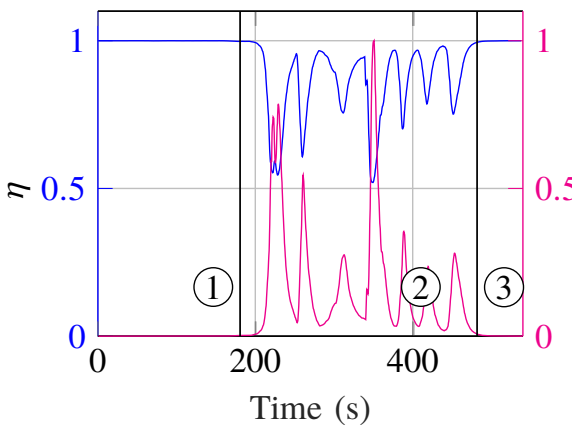

(a)

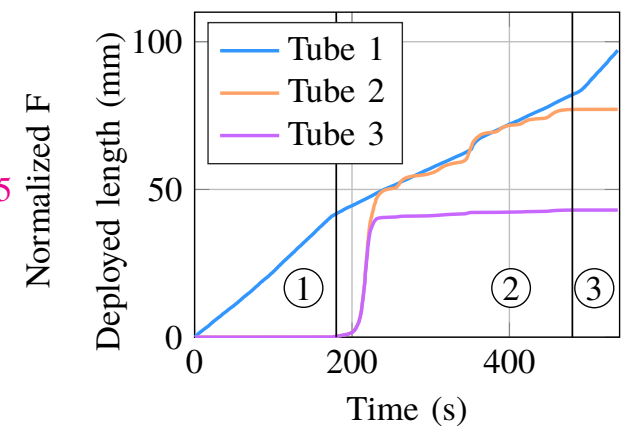

(b)

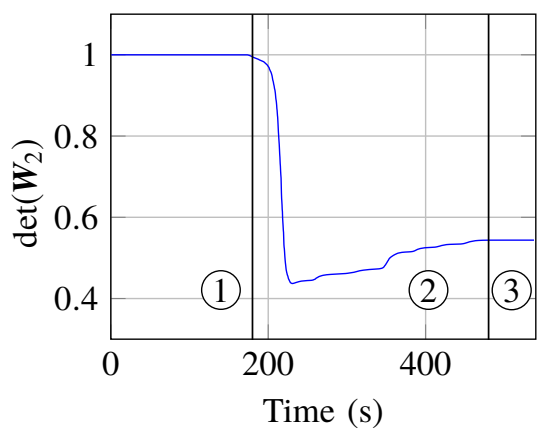

(c)

Fig. 6. (a) Normalized repulsive force $F$ (filtered) and $\eta$, (b) inserted tube lengths, and (c) stability of the CTR over time, with the deployment steps identified and labeled, in the case of the simulation with deployment in the nasal cavity.
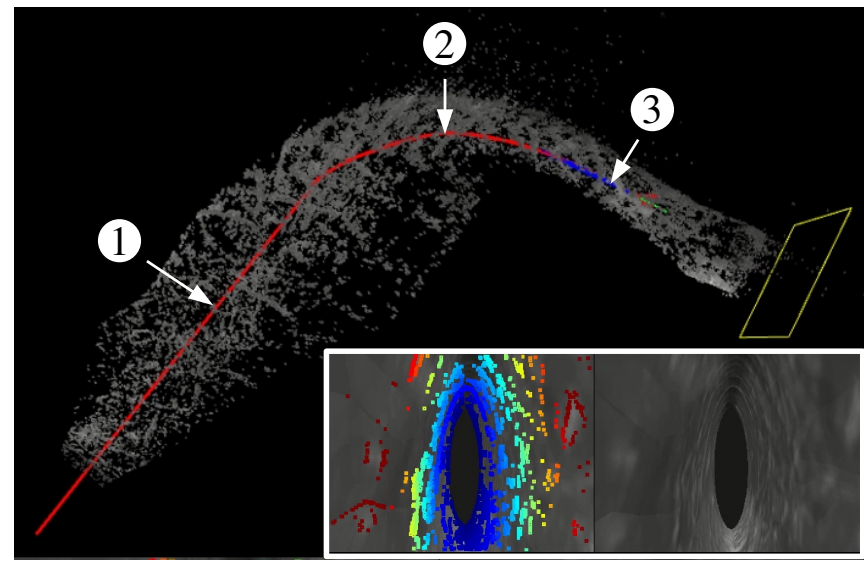

Fig. 7. Intranasal navigation in the simulated environment, with the deployment steps labeled. Top: reconstructed shape of nasal cavity, with the red lines showing the camera trajectory. Bottom: images coming from the virtual camera, with and without the detected features.

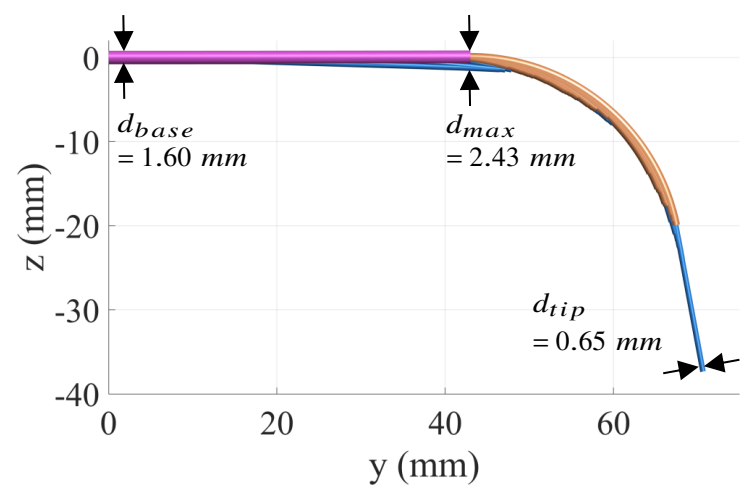

Fig. 8. Superposition of all intermediate CTR shapes adopted during deployment for the simulation, with measurements of the outer envelope. An approximate FTL deployment is observed.

by the CTR, used to compute a discrete FTL deployment error [27]. It illustrates the desired approximate FTL behavior, with an outer envelope slightly larger than the diameter of the largest tube. The validation using simulation on realistic anatomical data is thus satisfactory, and illustrates the interest and performance of the proposed approach.

\section{VALIDATION BY EXPERIMENT}

\section{A. Experimental Setup}

The performance of the proposed navigation approach is also assessed experimentally. The CTR actuation unit is composed of 3 motorized linear stages (stepper motors, LIMES 90-55-HSM, OWIS (Staufen, Germany)) for the tube translation. The main control software runs on a computer with GNU/Linux (Ubuntu 16.04). Another computer running Microsoft Windows 7 is used for communication with the actuation unit for compatibility reasons with the controllers. UDP socket is used for communication between the two computers. A low-cost miniature camera with a rolling shutter (Misumi MD-B802L-55) is attached at the extremity of tube 1 . The impact of its weight and the radial offset induced between the tube centerline and the sensor of the camera are neglected. The camera provides $640 \times 480$ pixels images at 25 images per second. Note that the camera parameters are very similar to those used on the virtual camera for the developed simulator, except for the shutter technology.

The characteristics of the CTR tubes are visible in Table III. They allow to have a straight, curved, and straight CTR shape after assembly. The environment of navigation is an origami tunnel with non-flat walls and a checkerboard-like image on the inner surface. Fig. 12 represents the origami tunnel with the robot during the navigation task. We opted for the artificial tunnel with chessboard markers because

TABLE III

CTR GEOMETRY FOR EXPERIMENTAL VALIDATION.

\begin{tabular}{lccc}
\hline Tube index & 1 & 2 & 3 \\
\hline Material & Nitinol & Nitinol & Nitinol \\
Inner diam $(\mathrm{mm})$ & 0.770 & 1.296 & 1.700 \\
Outer diam $(\mathrm{mm})$ & 1.020 & 1.520 & 2.000 \\
Curvature $\kappa_{i}\left(\mathrm{~mm}^{-1}\right)$ & 0.0000 & 0.0193 & 0.0064 \\
Total length $(\mathrm{mm})$ & 450.0 & 175.0 & 42.0 \\
\hline Links curvature: & & & \\
$\gamma_{1}=0.0000 \mathrm{~mm}^{-1}$ & $\circ$ & 0 & 0 \\
$\gamma_{2}=0.0150 \mathrm{~mm}^{-1}$ & $\circ$ & 0 & \\
$\gamma_{3}=0.0000 \mathrm{~mm}^{-1}$ & $\circ$ & & \\
\hline
\end{tabular}




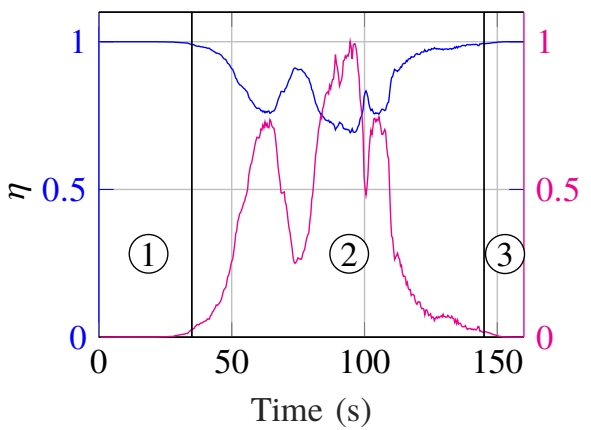

(a)

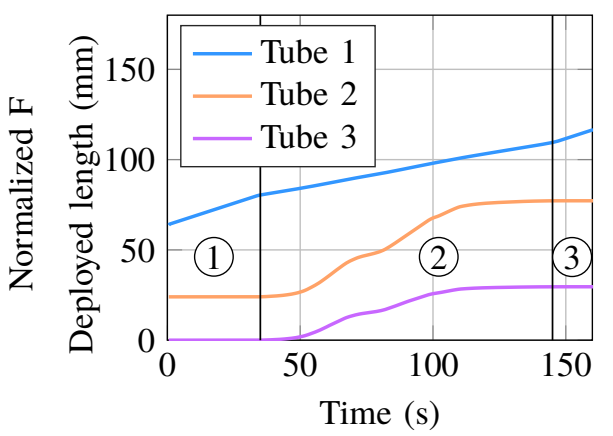

(b)

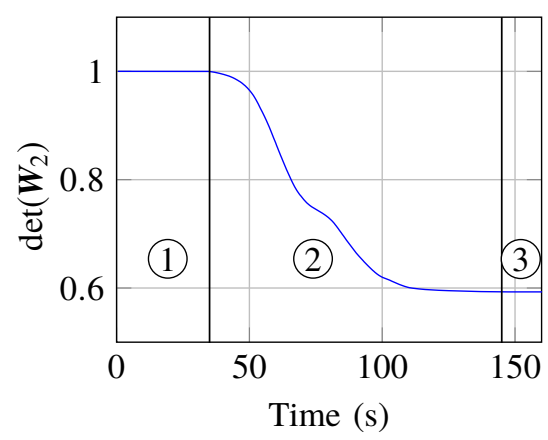

(c)

Fig. 9. (a) Normalized repulsive force $F$ (filtered) and $\eta$, (b) tube lengths inserted, and (c) stability of the CTR over time, with the deployment steps identified and labeled, in the case of the experiment with deployment in the origami tunnel.

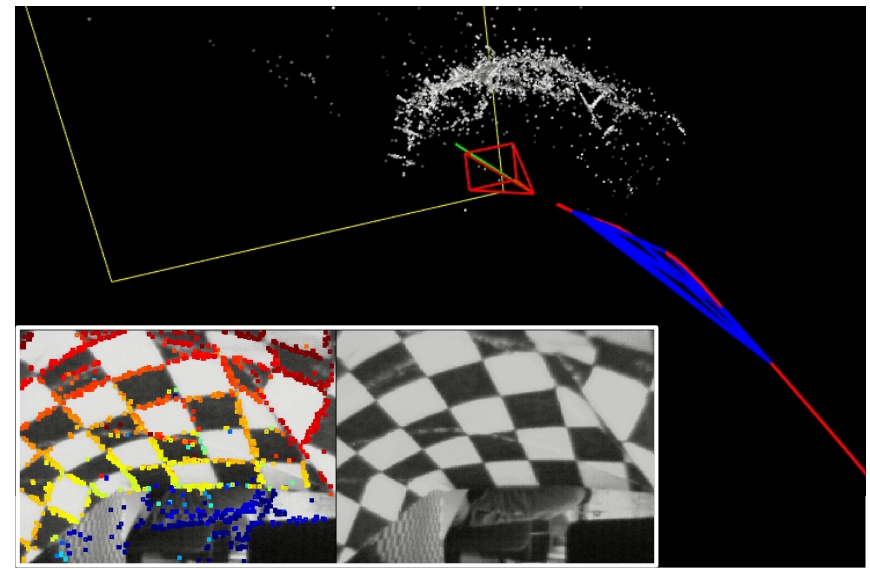

Fig. 10. Navigation in the origami tunnel. Top: reconstructed shape, with the red lines showing the camera trajectory. Bottom: images coming from the virtual camera, with and without the detected features.

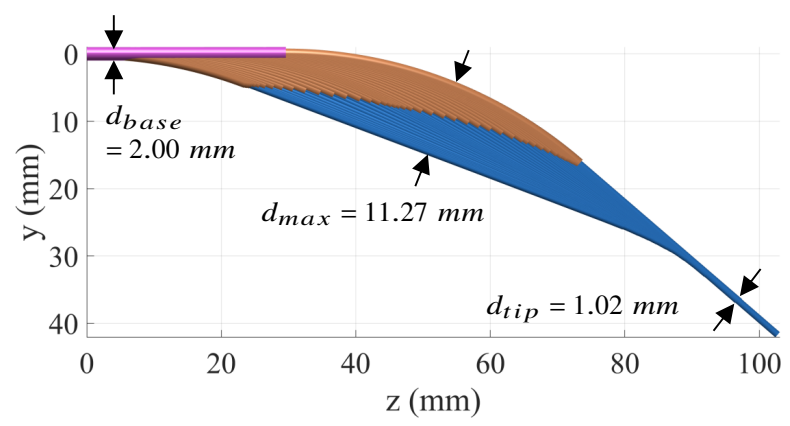

Fig. 11. Superposition of all the intermediate CTR shapes during deployment for the experiment, with measurements of the outer envelope.

of the performance of the miniature camera. The image quality is indeed low for standard components of one or two millimeters in size [22]. With such experiment, we can here validate the navigation approach in presence of image noise.

\section{B. Experimental Results}

The parameters used for the experimental validation are $\lambda_{T}=1, \lambda_{R}=1, v=0.5 \mathrm{~mm} \cdot \mathrm{s}^{-1}, d_{\pi}=30 \mathrm{~mm}, d_{\pi}^{*}=5 \mathrm{~mm}$, $d_{\mathcal{X}}^{\min }=10 \mathrm{~mm}, \delta=0.95, a=10$ and $\epsilon=0.1$. The CTR tip axis is initially roughly aligned with the tunnel. Due to limitations on the tube strokes, the experiment starts with the CTR partially deployed, so that its distal end is located in the tunnel. The experimental results presented in the following are illustrated in the provided video. The evolution of $\eta$ and $F$ over time are visible in Fig. 9a. The initially deployed lengths and their variation over time are visible in Fig. 9b. Three deployment steps can be identified on these figures, with the "Translating" task active during deployment steps 1, 2 and 3, and the "Rotating" task only active during deployment step 2. During deployment, the CTR remains stable, as visible in Fig. 9c, allowing the experiment to be fully conducted. The detection of key-points by the camera is successful, as visible in the bottom-left images of Fig. 10. The color of the key-points illustrates that their relative depth is correctly estimated, with the red points being close to the camera, and the blue ones being far from it. The top image of Fig. 10 shows the 3D point cloud faced by the camera during navigation, which is a part of the tunnel wall. Compared to the navigation in the simulated environment, the navigation is here assessed with inaccuracies in the reconstructed 3D point cloud of the environment. This can be observed with the scattering of the tunnel mapping in Fig. 10. Finally, Fig. 11 represents the superposition of all intermediate robot shapes during deployment. Larger displacements of the robot body compared to the simulation are visible. This is due to

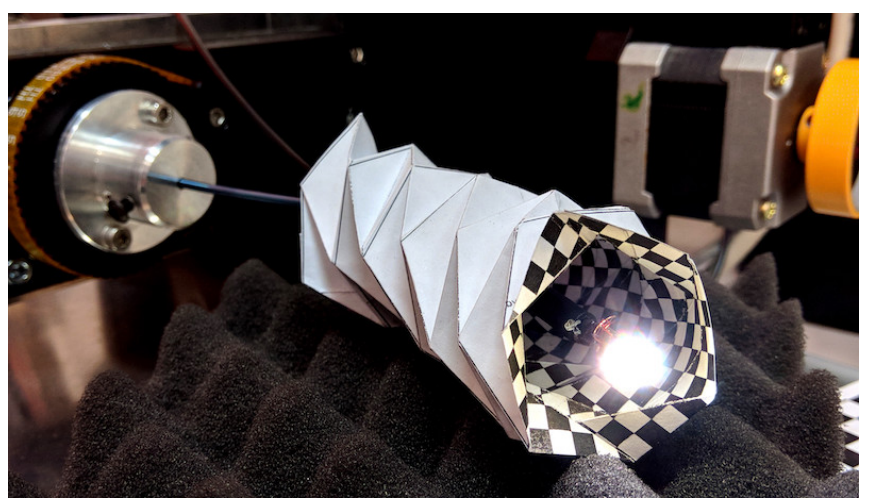

Fig. 12. CTR with the RGB camera attached at its distal end successfully navigating through the origami tunnel. 
the initial deployed lengths of the tubes, as our actuation system allowed a limited stroke for them. This lead to an angle between the CTR tip and the tunnel, that forced a reconfiguration of the CTR to avoid a collision at the tip. Such reconfiguration, that induces a CTR body motion, allows the tip of the robot to be tangent to the local curvature of the tunnel, which is the expected behavior during automatic navigation. Even in such sub-optimal conditions, the controller still manages to avoid contacts at the tip of the robot, with no contact observed either along the CTR body. The navigation is thus still successful with the robot crossing the tunnel, as visible in Fig. 12, so the experiment outlines the interest and robustness of the proposed approach.

\section{DISCUSSION AND CONCLUSION}

In this paper, an automatic intracorporeal navigation paradigm for CTR deployment was presented. The method combines an adapted monocular-based visual SLAM combined with a CTR that can follow the leader. It was shown that the proposed method is able to manage simultaneously the automatic navigation in an unknown environment, the collision avoidance, as well as the 3D mapping of the robot's environment, i.e., the anatomical structure. The proposed methods and materials were tested both in simulation, using a specifically-developed simulator using realistic anatomical data, and in an experimental scenario using a CTR with a camera mounted in an eye-in-hand configuration.

In the presented work, the CTR of interest has a trivial inverse kinematic model. Future developments will be focused on the extension to other CTRs that present redundancies, that can or cannot follow the leader, and more generally to the navigation of any continuum robot. Their entire shape could then be optimized based on the reconstructed environment acquired during deployment, to avoid collisions.

Further work will also be undertaken to improve the time-computation of the proposed method, the image quality (i.e., using higher quality miniature cameras) as well as the performance of the intracorporeal navigation method in more realistic conditions, i.e., in human cadavers. It is also foreseen to extend this method with other types of medical imaging probes such as intravascular ultrasound (IVUS) or optical coherence topography (OCT).

\section{REFERENCES}

[1] S. Ernst, F. Ouyang, C. Linder, K. Hertting, F. Stahl, J. K. Chun, H. Hachiya, D. Bansch, M. Antz, and K.-H. Kuck, Circulation, vol. 109 12, pp. 1472-1475, 2004.

[2] J. Burgner-Kahrs, D. C. Rucker, and H. Choset, "Continuum robots for medical applications: A survey," IEEE Trans. on Rob, vol. 31, no. 6, pp. 1261-1280, 2015.

[3] M. T. Chikhaoui, K. Rabenorosoa, and N. Andreff, "Kinematics and performance analysis of a novel concentric tube robotic structure with embedded soft micro-actuation," Mech. and Mach. Theory, vol. 104, pp. 234-254, 2016.

[4] P. Berthet-Rayne, G. Gras, K. Leibrandt, P. Wisanuvej, A. Schmitz, C. A. Seneci, and G. Z. Yang, "The i2snake robotic platform for endoscopic surgery," Ann. of Biomed. Eng., vol. 46, no. 10, pp. 16631675, 2018.

[5] H. B. Gilbert, J. Neimat, and R. J. Webster, "Concentric tube robots as steerable needles: Achieving follow-the-leader deployment," IEEE Trans. on Rob., vol. 31, no. 2, pp. 246-258, 2015.
[6] A. Garriga-Casanovas and F. R. y Baena, "Complete follow-the-leader kinematics using concentric tube robots," The Int. J. of Rob. Res., vol. 37, no. 1, pp. 197-222, 2018.

[7] A. Kuntz, L. G. Torres, R. H. Feins, R. J. Webster, and R. Alterovitz, "Motion planning for a three-stage multilumen transoral lung access system," in IEEE/RSJ Int. Conf. on Int. Rob. and Sys., 2015, pp. 32553261.

[8] L. Fichera, N. P. Dillon, D. Zhang, I. S. Godage, M. A. Siebold, B. I. Hartley, J. H. Noble, P. T. Russell, R. F. Labadie, and R. J. Webster, "Through the eustachian tube and beyond: A new miniature robotic endoscope to see into the middle ear," IEEE Rob. and Auto. Let., vol. 2, no. 3, pp. 1488-1494, 2017.

[9] G. Dwyer, F. Chadebecq, M. T. Amo, C. Bergeles, E. Maneas, V. Pawar, E. V. Poorten, J. Deprest, S. Ourselin, P. De Coppi, T. Vercauteren, and D. Stoyanov, "A continuum robot and control interface for surgical assist in fetoscopic interventions," IEEE Rob. and Auto. Let., vol. 2, no. 3, pp. 1656-1663, 2017.

[10] K. Wu, L. Wu, C. M. Lim, and H. Ren, "Model-free image guidance for intelligent tubular robots with pre-clinical feasibility study: Towards minimally invasive trans-orifice surgery," in IEEE Int. Conf. on Rob. and Auto., Aug 2015, pp. 749-754.

[11] A. V. Kudryavtsev, M. T. Chikhaoui, A. Liadov, P. Rougeot, F. Spindler, K. Rabenorosoa, J. Burgner-Kahrs, B. Tamadazte, and N. Andreff, "Eye-in-hand visual servoing of concentric tube robots," IEEE Rob. and Auto. Lett., vol. 3, no. 3, pp. 2315-2321, 2018.

[12] C. Girerd, T. Lihoreau, K. Rabenorosoa, B. Tamadazte, M. Benassarou, L. Tavernier, L. Pazart, E. Haffen, N. Andreff, and P. Renaud, "In vivo inspection of the olfactory epithelium: Feasibility of robotized optical biopsy," Ann. of Biomed. Eng., vol. 46, pp. 1951-1961, 2018.

[13] H. Durrant-Whyte and T. Bailey, "Simultaneous localization and mapping: part i," IEEE Rob. \& Aut. Mag., vol. 13, no. 2, pp. 99110, 2006.

[14] T. Bailey and H. Durrant-Whyte, "Simultaneous localization and mapping (slam): Part ii," IEEE Rob. \& Aut. Mag., vol. 13, no. 3, pp. 108-117, 2006.

[15] R. J. Webster III and B. A. Jones, "Design and kinematic modeling of constant curvature continuum robots: A review," The Int. J. of Rob. Res., vol. 29, no. 13, pp. 1661-1683, 2010.

[16] R. J. Hendrick, H. B. Gilbert, and R. J. Webster, "Designing snapfree concentric tube robots: A local bifurcation approach," in IEEE Int. Conf. on Rob. and Auto., 2015, pp. 2256-2263.

[17] Q. Peyron, K. Rabenorosoa, N. Andreff, and P. Renaud, "A numerical framework for the stability and cardinality analysis of concentric tube robots: Introduction and application to the follow-the-leader deployment," Mech. Mach. Theory, vol. 132, pp. 176 - 192, 2019.

[18] C. Bergeles, A. H. Gosline, N. V. Vasilyev, P. J. Codd, P. J. del Nido, and P. E. Dupont, "Concentric tube robot design and optimization based on task and anatomical constraints," IEEE Trans. on Rob., vol. 31, no. 1, pp. 67-84, 2015.

[19] J. Engel, V. Koltun, and D. Cremers, "Direct sparse odometry," IEEE transactions on pattern analysis and machine intelligence, vol. 40, no. 3, pp. 611-625, 2017.

[20] R. Mur-Artal, J. M. M. Montiel, and J. D. Tardos, "Orb-slam: a versatile and accurate monocular slam system," IEEE Trans. on Rob., vol. 31, no. 5, pp. 1147-1163, 2015.

[21] N. R. A., J. S. Lovegrove, and A. J. Davison, "Dtam: Dense tracking and mapping in real-time," in Int. Conf. on Comp. Vis., 2011, pp. 2320-2327.

[22] N. Yang, R. Wang, X. Gao, and D. Cremers, "Challenges in monocular visual odometry: Photometric calibration, motion bias, and rolling shutter effect," IEEE Rob. and Auto. Lett., vol. 3, no. 4, pp. 2878$2885,2018$.

[23] J. Engel, T. Schöps, and D. Cremers, "Lsd-slam: Large-scale direct monocular slam," in Europ. Conf. on Comp. Vis., 2014, pp. 834-849.

[24] O. Khatib, "Real-time obstacle avoidance for manipulators and mobile robots," in IEEE Int. Conf. on Rob. and Auto., 1985, pp. 500-505.

[25] S. Haddadin, R. Belder, and A. Albu-Schaeffer, "Reactive motion generation for robots in dynamic environments," in Int. Fed. of Auto. Cont. World Congress, 2011.

[26] C. Girerd, K. Rabenorosoa, P. Rougeot, and P. Renaud, "Towards optical biopsy of olfactory cells using concentric tube robots with follow-the-leader deployment," in IEEE/RSJ Int. Conf. on Int. Rob. and Sys., Sept. 2017, pp. 5661-5887.

[27] M. Neumann and J. Burgner-Kahrs, "Considerations for follow-theleader motion of extensible tendon-driven continuum robots," in IEEE Int. Conf. on Rob. and Auto., 2016, pp. 917-923. 